\title{
MODELAGEM GEOAMBIENTAL APLICADA À FAVORABILIDADE DE OCORRÊNCIA DE CARRAPATOS VETORES DE FEBRE MACULOSA NO MUNICÍPIO DE SEROPÉDICA, RJ
}

\author{
Andrea Kill Silveira \\ Universidade Federal Rural do Rio de Janeiro \\ BR-465, Km 7, Ecologia, Seropédica, Rio de Janeiro, 23890-000, Brasil \\ akillsilveira@gmail.com \\ Thiago Bernardo-Pedro \\ Universidade Federal Rural do Rio de Janeiro \\ BR-465, Km 7, Ecologia, Seropédica, Rio de Janeiro, 23890-000, Brasil \\ thiago.b.pedro@gmail.com
}

\begin{abstract}
RESUMO
Os carrapatos têm desempenhado papel importante na manutenção e transmissão de patógenos, entre animais silvestres, de produção e companhia e para humanos. Além disso, são importantes hospedeiros, vetores e amplificadores de patógenos, por estarem distribuídos por todo o planeta, com ampla diversidade de hospedeiros. A Análise Multicritério é um instrumento matemático que auxilia na comparação de diferentes cenários, fundamentados em muitos critérios, com a finalidade de conduzir os tomadores de decisão a uma escolha mais sensata. A modelagem de distribuição geográfica de espécies é uma técnica relativamente recente e tem se tornado uma ferramenta amplamente utilizada em estudos ecológicos e programas de conservação de espécies. Os modelos são também utilizados com sucesso para estudo de associações de variáveis com os agravos à saúde, na predição e controle de doenças. Dentro desse contexto, o objetivo do presente trabalho foi analisar a favorabilidade para a ocorrência de Amblyomma sculptum no município de Seropédica, RJ. A modelagem da distribuição espacial de carrapatos foi desenvolvida pelo Sistema de Análise Geoambiental SAGA/UFRJ da Universidade Federal do Rio de Janeiro. O método aplicado denomina-se Varredura Analítica e Integração Locacional, um procedimento diagnóstico de caráter ambiental. Para tal modelagem, três etapas foram necessárias: a definição das variáveis utilizadas, o processo de avaliação multicritério das variáveis e a assinatura ambiental. Para a geração do mapa de favorabilidade de ocorrência de carrapatos da espécie A. sculptum, utilizamos a Base de Dados Georreferenciados composta pelas seguintes variáveis: uso do solo e cobertura vegetal, favorabilidade de ocorrência de capivaras, geomorfologia e solo. O mapeamento classificatório das áreas com favorabilidade para a ocorrência de A. sculptum identificou que aproximadamente $87 \%$ do município de Seropédica foi favorável ou muito favorável para a ocorrência da espécie. As áreas com baixa favorabilidade se localizaram principalmente em locais com extrativismo mineral, intensa movimentação de caminhões, pouca vegetação, alta declividade e baixa favorabilidade para a ocorrência de capivaras. As áreas favoráveis ou muito favoráveis para a ocorrência de carrapatos localizaram-se, principalmente, em locais com pastagem e alta ou altíssima favorabilidade de ocorrência de capivaras. O presente trabalho pode contribuir para mostrar que a utilização de metodologias que envolvem modelos geoambientais podem ajudar no monitoramento da ocorrência de parasitos e hospedeiros.
\end{abstract}


Palavra-chave: Análise Multicritério; Distribuição Geográfica; Amblyomma sculptum; Rio de Janeiro.

\begin{abstract}
Ticks have developed an important role in the transmission of pathogens among wild animals, livestock, pets, and from them to humans. Besides that, ticks are important hosts, vectors and pathogen amplifiers, as they are spread worldwide and have a broad host diversity. The Multiple-criteria Decision Analysis is a mathematical tool that supports the comparison of different scenarios, grounded in many criteria, for the purpose of driving the decision makers to the best choice. The modeling of geographic distribution of species is a relatively recent technique that has become a tool widely applied to ecological studies and species conservation programs. The models are also successfully used to study the association between variables and health problems, to predict and control diseases. The aim of the present study was to analyze the favorability of Amblyomma sculptum occurrence in the municipality of Seropédica, RJ. The modeling was developed using the Geoenvironmental Analysis System SAGA/UFRJ, from Rio de Janeiro Federal University. The method applied is called Analytical Scan and Locational Integration, which is an environmental diagnostic procedure, and three steps were necessary: the variable definition, the multiple-criteria assessment and the environmental signature. To generate the map of favorability of A. sculptum occurrence, we used the Georeferenced Database composed by the following variables: land use and cover, favorability of capybara occurrence, geomorphology and type of soil. The classificatory mapping identified that around $87 \%$ of the municipality was favorable or very favorable for A. sculptum occurrence. The low favorability areas were especially related to mining, intense truck traffic, scarce vegetation, high declivity and low favorability of capybara occurrence. The favorable or very favorable areas for tick occurrence were especially related to pasture areas and high or very high favorability of capybara occurrence. The present work may contribute to show that the application of geo-environmental models can help monitoring the occurrence of hosts and parasites.
\end{abstract}

Keywords: Multiple-criteria Analysis; Geographic Distribution; Amblyomma sculptum; Rio de Janeiro.

\title{
Como Citar:
}

SILVEIRA, Andrea Kill; BERNARDO-PEDRO, Thiago. Modelagem Geoambiental Aplicada à Favorabilidade de Ocorrência de Carrapatos Vetores de Febre Maculosa no Município de Seropédica, RJ. In: SIMPÓSIO DE PESQUISA OPERACIONAL E LOGÍSTICA DA MARINHA, 19., 2019, Rio de Janeiro, RJ. Anais [...]. Rio de Janeiro: Centro de Análises de Sistemas Navais, 2019.

\section{INTRODUÇÃO}

O modelo atual de sociedade tem aumentado a demanda de recursos, acelerando a degradação ambiental e a fragmentação dos habitats. Por outro lado, nos últimos anos, em áreas urbanas e rurais, as ações de preservação dos recursos naturais, de seu uso sustentável e de atividades de turismo ecológico e rural propiciaram maior contato entre os organismos vivos dos ambientes silvestres, rurais e urbanos, inserindo a população humana na cadeia 
epidemiológica de algumas enfermidades, antes restritas aos ambientes naturais (BRADLEY; ALTIZER, 2007).

A diversidade, distribuição e abundância dos agentes patogênicos estão relacionadas às de seus hospedeiros, portanto o equilíbrio harmonioso da biodiversidade desempenha importante papel na distribuição e prevalência de doenças. Nos ecossistemas não perturbados a biodiversidade tende a ser maior e em equilíbrio. Em contraposição, em áreas perturbadas pode haver o domínio de uma ou poucas espécies generalistas, proporcionando o aumento de intensidade e a dispersão de patógenos específicos (SCHIMIDT; OSTFELD, 2001; ROSÀ; PUGLIESE, 2007; ESTRADA-PEÑA, 2009).

Neste contexto, os carrapatos têm desempenhado papel importante na manutenção e transmissão de patógenos, entre animais silvestres, de produção e companhia e para humanos. Os carrapatos são importantes hospedeiros, vetores e amplificadores de patógenos por estarem distribuídos por todo o planeta, com ampla diversidade de hospedeiros, e grande amplitude de nichos (ESTRADA-PEÑA, 2003; RANDOLPH, 2004; GUGLIELMONE et al., 2010). Para compreender melhor as interações entre os hospedeiros e os agentes patogênicos nos diferentes ecossistemas faz-se necessária a análise integradora de dados.

A Análise Multicritério é um instrumento matemático que auxilia na comparação de diferentes cenários, fundamentados em muitos critérios, com a finalidade de conduzir os tomadores de decisão a uma escolha mais sensata. Os métodos multicritério conjugam aspectos objetivos (quantitativos) e subjetivos (qualitativos), permitindo estruturar as possibilidades com uma gama de critérios de avaliação, cuja estrutura serve de apoio à tomada de decisão (PADILHA et al., 2014).

A principal finalidade do apoio à decisão multicritério é contribuir para a melhor saída que atenda inteiramente a todos os critérios. A principal regra, nesse caso, é que não há solução excelente, pois os critérios são conflituosos. O que há é uma alternativa que se adequa melhor às exigências do agente de decisão e que seja coerente com os critérios de avaliação que foram previamente estabelecidos (DIAS, 2015).

Por um lado, todos os critérios devem refletir alternativas de preferência, tão detalhadas quanto possível for; porém, por outro lado, o número de critérios deve ser o menor possível para que seja mais fácil operacionalizá-los. Desse modo, torna-se essencial alinhar a escolha e número de critérios com a escolha e número de alternativas (FRÖDELL, 2011).

Diversas técnicas utilizando os Sistemas Geográficos de Informação (SIG) têm sido utilizadas nas áreas ecológicas e epidemiológicas. Com a utilização do SIG é possível identificar, quantificar e investigar associações entre as variáveis envolvidas na ocorrência de fenômenos ambientais (XAVIER-DA-SILVA et al., 2001). A integração entre os métodos de análise multicritério e os SIG promove a sistematização e transformação de dados espaciais em uma resposta para a tomada de decisão. Nesse caso, o que determina as regras de decisão são as relações entre os dados espaciais (mapas de entrada) e os dados de saída (mapas finais) (MALCZEWSKI, 2004).

A modelagem de distribuição geográfica de espécies é uma técnica relativamente recente e tem se tornado uma ferramenta amplamente utilizada em estudos ecológicos e programas de conservação de espécies (PETERSON, 2006). Os modelos são também utilizados com sucesso para estudo de associações de variáveis com os agravos à saúde, na predição e controle de doenças (ESTRADA-PEÑA, 2003; LAMBIN et al., 2010; VANWAMBEKE et al., 2010). Segundo Lambin et al. (2010), os atributos associados ao ciclo de transmissão de patógenos estão relacionados com atributos da paisagem, padrão espacial e conectividade do habitat, vias de transmissão de patógenos entre vetores e hospedeiros, escala, uso do solo e ocupação e desenvolvimento humano. 
$\mathrm{Na}$ Europa e nos Estados Unidos, diversos modelos têm sido usados para verificar a distribuição de carrapatos e a possibilidade de estabelecimento de doenças zoonóticas. Em áreas rurais da Letônia, a modelagem através de SIGs foi utilizada para a identificação de fatores de risco na transmissão de encefalites transmitidas por carrapatos (TBE). As variáveis utilizadas foram as relacionadas com a característica da paisagem e o perfil socioeconômico. Os resultados evidenciaram que a TBE foi significativamente maior em áreas com maior proporção de florestas e seu entorno, os autores relacionaram estas áreas como as que têm maior abundância de carrapatos e seus hospedeiros (VANWAMBEKE et al., 2010).

$\mathrm{Na}$ Escócia, os fatores ambientais foram determinantes na abundância de ninfas de Ixodes ricinus e na incidência de Borrelia burgdorferi, como demonstrado por James et al. (2013). Os autores verificaram associação positiva dos carrapatos com a alta abundância de hospedeiros, como, veados e roedores. Também com floresta decídua e mista, quando comparado com florestas de coníferas, em áreas onde há o crescimento de vegetação gramínea e herbáceas, e locais que apresentam baixa umidade e altitude.

A modelagem para a verificação da extensão geográfica dos carrapatos Amblyomma sculptum e Ixodes, foi utilizada no México e Texas. Os autores utilizaram variáveis bioclimáticas e topográficas e percebeu-se que nesta região a espécie A. sculptum está relacionada às áreas de várzea, mangue, pântano, cerrado e floresta decídua, em locais com alta temperatura, e baixa altitude, principalmente entre $200 \mathrm{~m}$ e $1000 \mathrm{~m}$. Já Ixodes está associado a maiores altitudes, áreas com até 3600 m (ILLOLDI-RANGEL et al., 2012).

Atributos, como, tipo de solo, cobertura vegetal, altitude e distância de corpos d'água e de florestas foram utilizados por Bunnel et al. (2003) para analisar a distribuição espacial de Ixodes ricinus nos Estados Unidos. Os autores verificaram uma associação positiva entre a abundância de carrapatos e a distância de floresta decídua, corpos d'água, tipo de solo e altitude. $\mathrm{O}$ modelo identificou que áreas com solo arenoso, ou de alta granulometria, ou mesmo em locais com baixa altitude e moderada distância da floresta e corpos d'água representam fatores positivos para o risco de infestação por carrapatos I. ricinus. Os fatores protetores, ou seja, negativamente associados, são as áreas com altitude elevada, solos com silício e muito argilosos, áreas pantanosas e grande distância de corpos d'água e floresta.

A relação entre a presença de hospedeiros e carrapatos também foi estudada por Ferreira (2006) no município de Piracicaba/SP. A autora criou inicialmente um modelo preditivo de distribuição de capivaras e um mapa com a densidade de equinos no município. Com os mapas de ocorrência dos hospedeiros e outras variáveis, como características da paisagem, geomorfologia e climáticas, foi possível identificar áreas favoráveis para o desenvolvimento da população de A. sculptum e o risco para infestação humana por este carrapato. A autora sugere que as áreas ocupadas por pastagem, remanescentes de florestas e áreas em recuperação apresentam uma maior favorabilidade para o crescimento da população de $A$. sculptum. Indicou também que as áreas favoráveis estavam localizadas na periferia da cidade e que o aumento da favorabilidade esteve relacionado com o aumento da biomassa vegetal, associado ao aumento da temperatura.

Pedro (2015) utilizou modelagem de regressão para estudar a relação ente a distribuição de ocorrência de carrapatos $A$. sculptum adultos e diferentes características de paisagem, além da presença de hospedeiros, no município de Seropédica/RJ. O autor utilizou as seguintes variáveis explicativas: altitude, declividade, unidade geomorfológica, tipo de solo, presença de hospedeiros (equinos e capivaras) e favorabilidade de ocorrência de $A$. sculptum. Todas as variáveis foram consideradas estatisticamente significativas para a ocorrência de carrapatos, e declividade se mostrou como a variável independente de maior importância para a ocorrência de $A$. sculptum no município estudado.

O objetivo do presente trabalho foi analisar a favorabilidade para a ocorrência $A$. sculptum no município de Seropédica, RJ. 


\section{MATERIAL E MÉTODOS}

\subsection{DESCRIÇÃO DA ÁREA DE ESTUDO}

O município de Seropédica está localizado na região metropolitana do Rio de Janeiro, ocupa uma área de aproximadamente $284 \mathrm{~km}^{2}$ e população de 78.186 habitantes (IBGE, 2010). O clima, típico de regiões tropicais, com verões chuvosos e invernos secos, a precipitação média anual é de aproximadamente $1.200 \mathrm{~mm}$, a temperatura média anual de $25^{\circ} \mathrm{C}$ (MATTOS et al., 1999). O bioma da região é definido como de Floresta Atlântica, embora atualmente exista poucos fragmentos dessa vegetação (VELOSO et al., 1991). Parte da economia do município está relacionada à extração mineral, com destacada importância para a extração de areia, que é desenvolvida por um grande número de empresas, com mais de 80 cavas (ou lagoas) em atividade (MARQUES et al., 2012). Durante o processo histórico de ocupação dessa região houve uma fase de grande atividade agrícola, atualmente essa atividade é pequena e restrita a poucas culturas. A criação de gado é pequena, com aproximadamente 8 mil cabeças (LIMA; SANTOS, 1998; IBGE, 2006). A Universidade Federal Rural do Rio de Janeiro também contribui para o desenvolvimento urbano desta região e para economia, movimentando o comércio local (IBGE, 2010). Também há presença das Forças Armadas, neste município está localizado o Depósito Central de Munição do Exército Brasileiro (DCMun).

\subsection{MODELAGEM AMBIENTAL POR GEOPROCESSAMENTO}

Como a distribuição e abundância de carrapatos $A$. sculptum está diretamente relacionada com a presença de, pelo menos um de seus hospedeiros principais, como, equinos e/ou capivaras, tornou-se necessário inserção de dados sobre hospedeiros no modelo. Por falta de dados referentes à distribuição de equinos no município de Seropédica, optou-se pela modelagem sobre a favorabilidade de ocorrência de capivaras, para dar apoio ao modelo favorabilidade de ocorrência do carrapato (SILVEIRA; FONSECA, 2013).

A modelagem da distribuição espacial de carrapatos foi desenvolvida pelo Sistema de Análise Geoambiental SAGA/UFRJ da Universidade Federal do Rio de Janeiro (LAGEOP, 2007). O método aplicado denomina-se Varredura Analítica e Integração Locacional, um procedimento diagnóstico de caráter ambiental (XAVIER-DA-SILVA et al., 2001). Para tal modelagem, três etapas foram necessárias, sendo, a definição das variáveis utilizadas, o processo de avaliação multicritério das variáveis e a assinatura ambiental.

Realizou-se um inventário ambiental, apresentando cartograficamente as variáveis consideradas relevantes para a favorabilidade de ocorrência de carrapatos (SZABO et al., 2009; VERONEZ et al., 2010; QUEIROGAS et al., 2012; SILVEIRA; FONSECA, 2013). Gerou-se, então, um conjunto de mapas temáticos o qual foi denominado Base de Dados Georreferenciados (BDG) para carrapatos.

A BDG foi composta pelas variáveis, (1) uso de solo e cobertura e vegetal, (2) favorabilidade de ocorrência de capivaras, (3) geormorfologia, (4) solo e (5) declividade. Todos os mapas com resolução espacial de $25 \mathrm{~m}$ e escala cartográfica de 1: 50 000, georreferenciados na projeção Universal Transverse Mercator (UTM), fuso 23 Sul, Datum SAD69.

Com a BDG definida, procedeu-se a definição e avaliação das áreas com potencial para a ocorrência de carrapatos no município de Seropédica, RJ. Foi utilizado o procedimento de avaliação multicritério, fundamentado na experiência profissional de membros da equipe de trabalho, bem como por consulta a literatura científica (XAVIERDASILVA, 2001). Para a avaliação criou-se, então, uma árvore de gestão espacializada, onde os mapas temáticos foram organizados de acordo com a importância para a ocorrência 
do evento. Esses mapas foram sendo combinados em todos os níveis, até gerar uma síntese avaliativa do produto da integração de todos os mapas constituintes da árvore de decisão. Após a atribuição dos pesos aos parâmetros ambientais da BDG, distribuída na árvore de gestão espacializada, foi elaborada uma "Planilha de Valoração", com as notas das classes dos mapas temáticos. Portanto, a cada variável é atribuído um percentual que varia de $0 \mathrm{a}$ $100 \%$. E para cada categoria e atribuída uma nota, na escala de 0 a 10 . Estas notas devem representar a possibilidade de ocorrência do fenômeno em avaliação, nas diferentes áreas geográficas relativas a cada categoria apresentada nos mapas temáticos.

O algoritmo utilizado gera um valor para cada elemento da matriz A pela multiplicação do valor da avaliação geral de cada mapa, que e o seu peso (parcela do intervalo entre 0 e 1) pelo valor (nota) relativo a categoria da legenda do mapa que tenha sido atribuído a cada elemento da matriz A. Com a formulação, a seguir e criada a avaliação de cada elemento da matriz e, consequentemente, o mapeamento avaliativo final:

$$
\mathrm{A}_{\mathrm{i}, \mathrm{j}}=\Sigma_{\mathrm{k}=1}^{\mathrm{n}}\left(\mathrm{P}_{\mathrm{ij}(\mathrm{k})} \cdot \mathrm{N}_{\mathrm{ij}(\mathrm{k})}\right) \text {, onde: }
$$

$A_{i, j}=$ possibilidade de ocorrência do evento analisado no elemento (pixel) i,j da matriz (mapa) resultante;

$\mathrm{n}$ = número de variáveis (mapas) utilizadas;

$\mathrm{P}_{\mathrm{ij}(\mathrm{k})}=$ peso (percentual) da contribuição do parâmetro " $\mathrm{k}$ ", em relação aos demais, para a ocorrência do evento analisado;

$\mathrm{N}_{\mathrm{ij}(\mathrm{k})}=$ nota, segundo os avaliadores, dentro da escala de 0 a 10 , da ocorrência do evento analisado, na presença da classe encontrada na linha $\mathrm{i}$, coluna $\mathrm{j}$ do mapa $\mathrm{k}$.

Por fim, os mapas temáticos foram submetidos ao procedimento de Assinatura Ambiental que corresponde a uma investigação empírica por varredura das características ambientais com maior probabilidade de influenciar no fenômeno analisado, gerando um conjunto de tabelas que mostram a matriz das assinaturas que constam nos resultados. Esta etapa fornece apoio e segurança aos procedimentos avaliativos que definem a situação ambiental.

\subsubsection{Modelagem Geoambiental para a Favorabilidade de Ocorrência de A. sculptum}

Para a geração do mapa de favorabilidade de ocorrência de carrapatos da espécie $A$. sculptum utilizamos a BDG composta pelas variáveis, uso do solo e cobertura e vegetal, favorabilidade de ocorrência de capivaras, geomorfologia e solo. A árvore de gestão espacializada para a adoção de pesos está descrita na Tabela 1. E as notas são mostradas na Tabela 2.

Tabela 1. Árvore de gestão espacializada para a adoção de pesos para a modelagem da favorabilidade de ocorrência de Amblyomma sculptum no município de Seropédica, RJ.

\begin{tabular}{|c|c|}
\hline Variáveis & Pesos $(\%)$ \\
\hline Uso do solo e Cobertura Vegetal & 32 \\
\hline Favorabilidade de ocorrência de capivaras & 30 \\
\hline Geomorfologia & 15 \\
\hline Solo & 13 \\
\hline Declividade & 10 \\
\hline Total & 100 \\
\hline
\end{tabular}


Tabela 2. Notas atribuídas às categorias que compõem os mapas utilizados na análise de favorabilidade de ocorrência de Amblyomma sculptum no município de Seropédica, RJ.

\begin{tabular}{|c|c|c|c|}
\hline \multirow{3}{*}{$\begin{array}{l}\text { Variáveis } \\
\text { Uso do solo } \\
\text { cobertura vegetal }\end{array}$} & \multicolumn{3}{|c|}{ Categorias } \\
\hline & \multirow[b]{2}{*}{$\begin{array}{l}\text { 1-3 } \\
\text { eÁreas alagáveis/Área } \\
\text { urbana/Área industrial } \\
\text { /Afloramento rocha/Solo } \\
\text { exposto/ } \\
\text { Extrativismo mineral }\end{array}$} & \multirow{2}{*}{$\begin{array}{c}4-7 \\
\text { Cultivo/Eucalipto }\end{array}$} & $8-10$ \\
\hline & & & $\begin{array}{l}\text { Pastagem/ Vegetação } \\
\text { herbácea } \\
\text { arbustiva/Fragmento de } \\
\text { vegetação arbórea/ }\end{array}$ \\
\hline Declividade & $>40 \%$ & $11-40 \%$ & $0-10 \%$ \\
\hline Geomorfologia & $\begin{array}{l}\text { Encostas de Talus/Borda } \\
\text { dissecada de planalto }\end{array}$ & $\begin{array}{l}\text { Rampas de } \\
\text { colúvio/Patamar } \\
\text { dissecado em colinas e } \\
\text { vales } \\
\text { estruturais/Planície } \\
\text { flúvio lacruste deltaica }\end{array}$ & $\begin{array}{l}\text { Colinas/Planície colúvio- } \\
\text { aluviar/ } \\
\text { aluvionar de cobertura/ }\end{array}$ \\
\hline Tipo de solo & Neossolo flúvico & $\begin{array}{l}\text { Associação entre } \\
\text { cambissolo e argissolo } \\
\text { vermelho-amarelo } \\
\text { distrófico/Associação } \\
\text { entre argissolo } \\
\text { vermelho-amarelo } \\
\text { distrófico, cambissolo e } \\
\text { Argissolo vermelho } \\
\text { distrófico/Plintossolo/ } \\
\text { Gleissolo/Organossolo/ } \\
\text { Neossolo }\end{array}$ & $\begin{array}{l}\text { Associação entre } \\
\text { cambissolo e neossolo } \\
\text { litólico/Planossolo }\end{array}$ \\
\hline $\begin{array}{l}\text { Favorabilidade } \\
\text { Capivara }\end{array}$ & & $\begin{array}{l}\text { Baixa e Moderada } \\
\text { favorabilidade }\end{array}$ & $\begin{array}{l}\text { Alta e } \\
\text { favorabilidade }\end{array}$ \\
\hline
\end{tabular}

Para a categorização final do mapa, as notas de 3 até 5 foram consideradas pouco favoráveis, as notas 6 e 7 favoráveis, e as notas 8 e 9 como muito favoráveis.

\section{RESULTADOS E DISCUSSÃO}

\subsection{ESTUDO DA GEODIVERSIDADE AMBIENTAL PARA O MUNICÍPIO DE SEROPÉDICA, RJ}

As assinaturas realizadas nos mapas representativos das variáveis contidas neste estudo revelaram que o município de Seropédica possui área de pastagem equivalente a 7 828 ha (27\%), o cultivo ocupa uma área de 4229 ha (16\%) a área com fragmento de vegetação arbóreo corresponde a 2885 ha (11\%). O solo predominante é o planossolo (42\%) seguido do argissolo vermelho-amarelo distrófico (27\%). E as feições geomorfológicas predominantes são as planícies colúvio-aluvionar (32\%) e aluvionar de cobertura (21\%) que fazem parte do sistema geomorfológico definido como Baixada de Sepetiba e também uma área de borda dissecada de planalto estrutural (13\%) que faz parte do sistema de Encosta da Serra do Mar. A declividade de $0-2,5 \%$ ocupa $52 \%$ de todo o município indicando a característica de baixada. 


\subsection{MODELAGEM GEOAMBIENTAL PARA A FAVORABILIDADE OCORRÊNCIA DE A. SCULPTUM NO MUNICÍPIO DE SEROPÉDICA, RJ}

O mapeamento classificatório das áreas com favorabilidade para a ocorrência de $A$. sculptum identificou que aproximadamente $87 \%$ do município de Seropédica foi favorável ou muito favorável para a ocorrência da espécie (Tabela 3 ).

Tabela 3. Valores obtidos pela assinatura ambiental indicando os graus de favorabilidade e as categorias mais relevantes relacionadas à ocorrência de Amblyomma sculptum no município de Seropédica, RJ.

\begin{tabular}{|c|c|c|c|c|c|c|}
\hline \multicolumn{2}{|c|}{ Favorabilidade } & \multicolumn{5}{|c|}{ Variáveis } \\
\hline & $\begin{array}{c}\text { Área em ha } \\
(\%)\end{array}$ & $\begin{array}{l}\text { Declividade } \\
(\%)\end{array}$ & $\begin{array}{l}\text { Solo } \\
(\%)\end{array}$ & $\begin{array}{c}\text { Geomorfologia } \\
(\%)\end{array}$ & $\begin{array}{c}\text { Favorabilidade } \\
\text { ocorrência de capivaras }\end{array}$ & $\begin{array}{l}\text { Uso do solo e } \\
\text { cobertura vegetal }\end{array}$ \\
\hline$\overline{\text { Baixa }}$ & $\begin{array}{l}2949 \\
(13 \%)\end{array}$ & $\begin{array}{c}20-40 \mathrm{~m} \\
(43 \%)\end{array}$ & $\begin{array}{l}\text { Planossolo } \\
\quad(31 \%)\end{array}$ & $\begin{array}{c}\text { Borda dissecada } \\
\text { de planalto } \\
\text { estrutural } \\
(48 \%)\end{array}$ & $\begin{array}{l}\text { Baixa favorabilidade } \\
(58 \%)\end{array}$ & $\begin{array}{l}\text { Extrativismo } \\
\text { mineral } \\
(44 \%)\end{array}$ \\
\hline Favorável & $\begin{array}{l}10052 \\
(46 \%)\end{array}$ & $\begin{array}{c}0-2,5 \mathrm{~m} \\
(43 \%)\end{array}$ & $\begin{array}{c}\text { Argissolo } \\
\text { Vermelho- } \\
\text { Amarelo } \\
\text { Distrófico } \\
(29 \%) \\
\text { Planossolo } \\
(28 \%)\end{array}$ & $\begin{array}{l}\text { Planície colúvio- } \\
\text { aluvionar } \\
(25 \%) \\
\text { Borda dissecada } \\
\text { de planalto } \\
\text { estrutural } \\
(19 \%)\end{array}$ & $\begin{array}{c}\text { Moderada } \\
\text { favorabilidade } \\
(34 \%) \\
\text { Alta favorabilidade } \\
(27 \%)\end{array}$ & $\begin{array}{l}\text { Cultivo e } \\
\text { propriedades } \\
\text { rurais } \\
(31 \%) \\
\text { Pastagem } \\
(20 \%)\end{array}$ \\
\hline Muito & $\begin{array}{l}8878 \\
(41 \%)\end{array}$ & $\begin{array}{l}0-2,5 \mathrm{~m} \\
(61 \%)\end{array}$ & $\begin{array}{l}\text { Planossolo } \\
\quad(54 \%)\end{array}$ & $\begin{array}{l}\text { Planície colúvio- } \\
\text { aluvionar } \\
(54 \%)\end{array}$ & $\begin{array}{c}\text { Alta favorabilidade } \\
(67 \%) \\
\text { Altíssima favorabilidade } \\
(23 \%)\end{array}$ & $\begin{array}{l}\text { Pastagem } \\
(57 \%) \\
\text { Vegetação } \\
\text { herbácea- } \\
\text { arbustiva } \\
(20 \%)\end{array}$ \\
\hline
\end{tabular}

A representação da favorabilidade de ocorrência de $A$. sculptum é apresentada na Figura 1. 


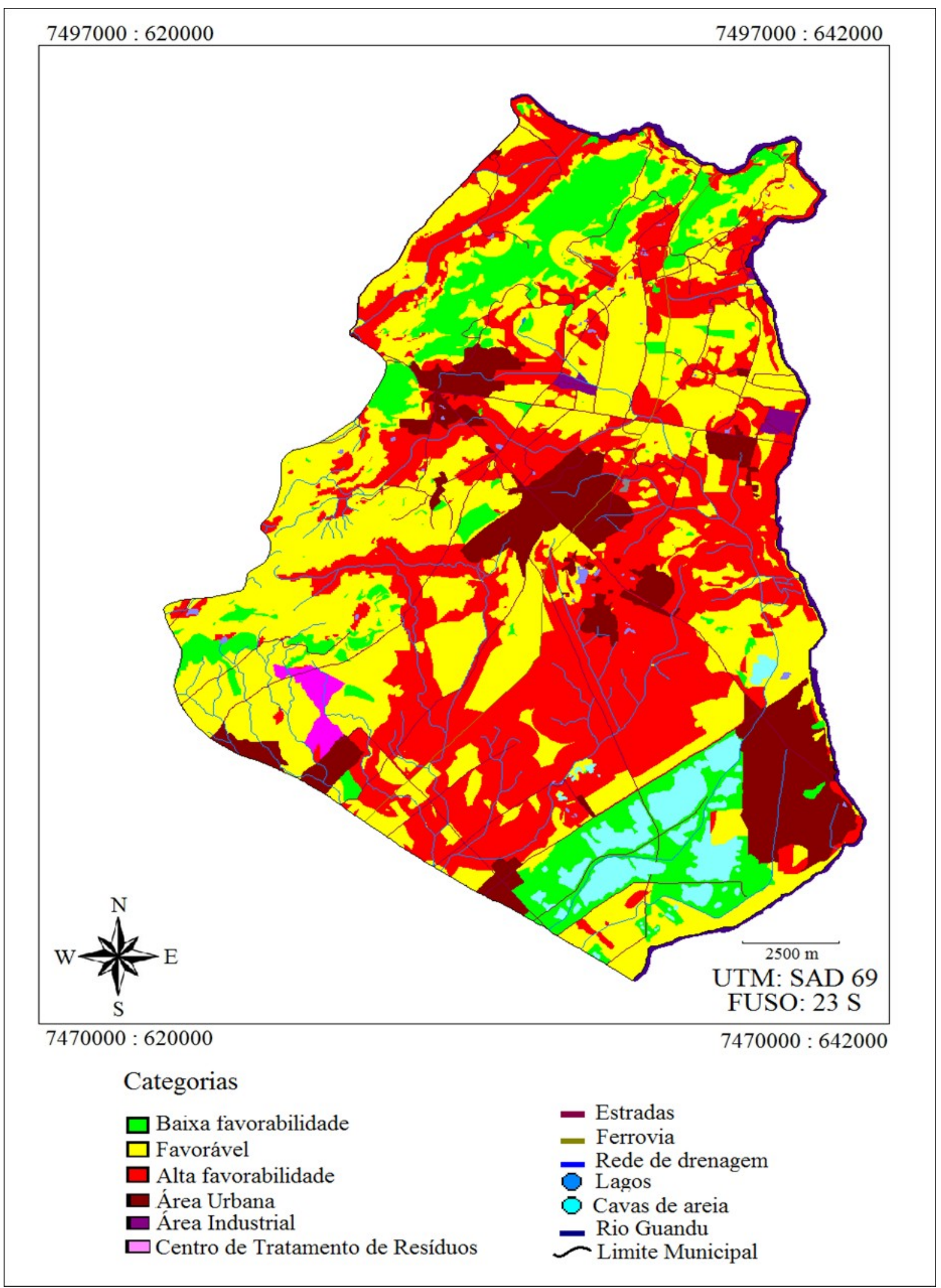

Figura 1. Representação da favorabilidade de ocorrência de Amblyomma sculptum no município de Seropédica, RJ. 
As áreas com baixa favorabilidade se localizaram principalmente em locais com extrativismo mineral, intensa movimentação de caminhões, pouca vegetação, alta declividade e baixa favorabilidade para a ocorrência de capivaras. Embora A. sculptum seja um carrapato frequentemente coletado em áreas antropizadas, e também generalista quanto ao hospedeiro, algumas exigências para seu ciclo de vida são necessárias, ou seja, no presente trabalho as áreas identificadas como de baixa favorabilidade não apresentam vegetação suficiente para a manutenção dos estádios de vida dos carrapatos e também não há possibilidade da presença de hospedeiros, características indicadas por Szabó et al. (2009) e Veronez et al. (2010) como importantes para a presença de carrapatos numa determinada área.

Já as áreas favoráveis para a ocorrência de carrapato foram indicadas em locais de pastagem e propriedades agrícolas com alta, ou altíssima favorabilidade de ocorrência de capivaras. Essas áreas possivelmente podem manter populações de carrapatos, pois segundo Labruna et al. (2001) a presença de pastagem é uma variável importante para a manutenção da população de A. sculptum. E hospedeiros como equinos, bovinos e capivaras podem frequentar o local. De acordo com os achados de Cançado et al. (2008) e Szabó et al. (2009) os animais citados acima são hospedeiros potenciais para aquela espécie de carrapato. As áreas de cultivo também foram indicadas como favoráveis. Nestes locais pode ocorrer a presença de capivaras, que podem carrear carrapatos. Entretanto, segundo Souza et al. (2009) e Queirogas et al. (2012) sem a frequência dos hospedeiros, a população de carrapatos também não se estabelecerá.

As áreas muito favoráveis para a ocorrência de carrapatos localizam-se, principalmente, em locais com pastagem e alta ou altíssima favorabilidade de ocorrência de capivaras. A verificação de que áreas de pastagem mal manejadas é altamente favorável para a ocorrência de carrapatos foi relatada por Labruna et al. (2001) e Silveira e Fonseca (2013). E o município de Seropédica possui uma grande área de pastagem em seu território, essa pastagem é ocupada por bovinos e equinos (IBGE, 2006).

A categoria de vegetação herbácea arbustiva também teve destacada importância para a ocorrência de carrapatos. A assinatura deste tipo de vegetação foi contrastada com a favorabilidade de capivaras (SILVEIRA, 2014), pois verificou-se que 65\% dela está em áreas com alta ou altíssima favorabilidade para a ocorrência de capivaras, ou seja, tem-se neste tipo de cobertura vegetal, assim como nas áreas com gramínea alta ou pastagem mal manejada, além da presença de hospedeiro, a formação vegetal que forma um tampão absorvendo a radiação solar durante o dia, reduzindo a perda de calor durante a noite e mantendo a umidade relativa. Assim, atuam diretamente no desenvolvimento e sobrevivência dos estádios de vida livre e regulam a disponibilidade de estádios evolutivos nos diferentes ambientes (SONENSHINE, 1993).

Uma variável importante citada por Bunnel et al. (2003) para a ocorrência de $I$. ricinus é a distância de florestas e corpos d'água. No presente trabalho não utilizamos essas variáveis. Mas elas estão intimamente relacionadas à favorabilidade de capivaras. A capivara é hospedeiro primário de $A$. sculptum, e já foi confirmado que esta espécie de hospedeiro mantém o carrapato no município de Seropédica (Silveira; Fonseca, 2013). Em locais próximos às áreas de mata e/ou capoeira e também cursos d'água, onde há presença de capivaras, há um grande número de carrapatos. A observação de que áreas de capoeira, vegetação densa e áreas de ecótonos são favoráveis para a ocorrência de carrapatos, também foi verificada por Ferreira (2006), Estrada-Peña et al. (2004) e Vanwambeke et al. (2010).

De acordo com os achados de Pedro et al. (2016), planossolo é o tipo de solo que mais favorece a ocorrência de carrapatos $A$. sculptum adultos no município de Seropédica, pois, segundos os autores, este tipo de solo permite que o microclima próximo à superfície permaneça mais úmido, o que facilita aos carrapatos manterem a hidratação. Ainda segundo 
Bunnel et al. (2003), a espécie de carrapato I. ricinus, nos Estados Unidos, tem associação positiva com solo arenoso ou de alta granulometria. No presente trabalho, verifica-se que as áreas com alta favorabilidade de ocorrência de A. sculptum estão em solo classificado como planossolo, este tipo de solo é caracterizado como arenoso, concordando com os dados apresentados para I. ricinus. Vale destacar que a classificação pouco favorável também é caracterizada por solo planossolo, mas a combinação de todas as variáveis daquela classe não favorece o desenvolvimento do carrapato. São vários os fatores que contribuem para o desenvolvimento e estabelecimento de uma população e não apenas um fator isolado (ILLOLDI-RANGEL et al., 2012).

O modelo indicou que o município de Seropédica tem grande favorabilidade para a ocorrência de carrapatos. É importante destacar que o município está num processo acelerado de expansão urbana, com incentivo à industrialização do município e de municípios vizinhos como Itaguaí e Paracambi, levará a um aumento populacional em Seropédica. Normalmente, o aumento da população se dá em áreas periféricas, onde podem ocorrer diversos problemas com o aumento de carrapatos. Em Piracicaba-SP, a área de maior risco de infestação por carrapatos são as áreas periféricas do município, no entorno da área urbana (FERREIRA, 2006). Vanwambeke et al. (2010) também observou que nas áreas rurais da Letônia, onde a população possui escolaridade de até 4 anos, o risco de TBE (Encefalite Transmitida por Carrapatos) foi maior. Dantas-Torres et al (2012) verificaram que fragmentos florestais proporcionam um ambiente favorável para carrapatos e pequenos mamíferos, que são altamente expostas a rickettsias. Também indicam que fragmentos florestais em áreas rurais são normalmente associados com maior diversidade de pequenos mamíferos, assim como taxas de densidade de carrapatos mais elevados.

Há uma relação entre a presença de hospedeiros e carrapatos, essa relação já foi relatada por Szabó et al. (2007) e Veronez et al. (2010) no bioma do cerrado e por Szabó et al. (2009) na Mata Atlântica. Segundo o Ministério da Saúde (BRASIL, 2019) três casos de febre maculosa foram confirmados no município de Seropédica, sendo 2 deles no ano de 2007 e 1 no ano de 2017. Embora não seja possível identificar em qual ambiente houve a infecção, ou se os casos foram autóctones, há necessidade de monitoramento constante da ocorrência de capivaras, importante hospedeiro de A. sculptum e hospedeiro amplificador da bactéria $R$. rickettsii agente etiológico da FMB.

A área onde se localiza o DCMUN está localizada numa região com alta favorabilidade de ocorrência de carrapatos, neste local a presença constante de capivaras, além de cavalos. Silveira e Fonseca (2013) coletaram uma grande quantidade de carrapatos A. sculptum no DCMun. Esta área possui características que favorecem a presença de carrapatos desta espécie, como a formação de mosaicos de paisagens, geralmente com gramínea alta e/ou outras herbáceas e arbustos, e proximidade com área de capoeira e/ou fragmento de mata secundária e/ou reflorestamento. Esses ambientes, além de fornecerem um microclima adequado para o desenvolvimento de A. sculptum, também fornecem abrigo e alimento para os hospedeiros, como equinos, bovinos e principalmente capivaras, registrados nestes locais durante as coletas. Importante salientar o que os militares, deste e de outros batalhões, utilizam a área para atividades de treinamento militar, o que deixa essa população exposta ao contato direto com os carrapatos. Ainda segundo Silveira e Fonseca (2013) os estádios imaturos, mais generalistas quanto aos hospedeiros, estão presentes principalmente nos meses mais secos do ano. Uma amostra de larvas do carrapato Amblyomma positiva para a presença de bactérias do gênero Rickettsia spp. foi encontrada no DCMUN na estação do outono, numa área de bambuzal (SILVEIRA; FONSECA, 2013), enfatizando a necessidade de monitoramento da população que frequenta o local e exigindo, assim, maior cuidado dos frequentadores deste local, nesta época do ano. 
Com a análise geoambiental foi possível concluir que o município de Seropédica, RJ, tem ampla área de favorabilidade de ocorrência de carrapatos. Tal informação poderá contribuir para um amplo espectro de ações e intervenções antrópicas referentes ao habitat e ao perfil de comportamento da referida espécie. Então, o presente trabalho pode contribuir para mostrar que a utilização de metodologias que envolvem modelos geoambientais podem ajudar no monitoramento da ocorrência de parasitos e hospedeiros. Com estes modelos, os recursos financeiros investidos para o controle e erradicação de doenças podem ser devidamente alocados, sem o desperdício de dinheiro e força de trabalho.

\section{REFERÊNCIAS BIBLIOGRÁFICAS}

[1] BRADLEY, C.A.; ALTIZER, S. Urbanization and the ecology of wildlife diseases. Trends in Ecology \& Evolution, v. 22, p. 95-102, 2007.

[2] BRASIL. Ministério da Saúde. Febre Maculosa - Casos confirmados notificados no sistema de informação de agravos de notificação - Sinan. Disponível em: http://dtr2004.saude.gov.br/sinanweb/tabnet/dh?sinan/fmaculosa/bases/

febremaculosabr.def. Acesso em: 29 abr. 2019.

[3] BUNNEL, J.E.; PRICE, S.D.; DAS, A.; SHIELDS, T.M.; GLASS, G.E. Geographic information systems and spatial of adult Ixodes scapularis (Acari: Ixodidae)c in the middle Atlantic Region of the U.S.A. Journal of Medical Entomology, v. 40, n. 4, p. 570-576, 2003.

[4] CANÇADO, P.H.D.; PIRANDA, E.M.; MOURÃO, G.M.; FACCINI, J.L.H. Spatial distribution and impact of cattle-raising on ticks in the Pantanal region of Brazil by using the CO2 tick trap. Parasitology Research, v. 103, n. 2, p. 371-377, 2008.

[5] DANTAS-TORRES, F.; ALÉSSIO, F.M.; SIQUEIRA, D.B.; MAUFFREY, J.F.; MARVULO, M.F.V.; MARTINS, T.F.; MORAES-FILHO, J.; CAMARGO, M.C.G.O.; D'AURIA, S.R.N.; LABRUNA, M.B.; SILVA, J.C.R. Exposure of small mammals to ticks and rickettsiae in Atlantic Forest patches in the metropolitan area of Recife, north-eastern Brazil. Parasitology, v. 139, n. 1, p. 83-91, 2012.

[6] DIAS, E.M.P.S. Modelo de apoio à decisão multicritério para seleção de fornecedores de azeite. 2015, 56 f. Dissertação (Mestrado em Modelação, Análise de Dados e Sistemas de Apoio à Decisão) - Faculdade de Economia, Universidade do Porto, Porto, 2015.

[7] ESTRADA-PEÑA, A. Diluting the dilution effect: a spatial Lyme model provides evidence for the importance of habitat fragmentation with regard to the risk of infection. Geospatial Health, v. 3, p. 143-155, 2009.

[8] ESTRADA-PEÑA, A.; GUGLIELMONE, A.A.; MANGOLD, A.J. The distribution and ecological 'preferences' of the tick Amblyomma sculptum (Acari: Ixodidae), an ectoparasite of humans and other mammals in the Americas. Annals of Tropical Medicine \& Parasitology, v. 98, n. 3, p. 283-292, 2004.

[9] ESTRADA-PEÑA, A. The relationships between habitat topology, critical scales of connectivity and tick abundance Ixodes ricinus in a heterogeneous landscape in northern Spain. Ecography, v. 26, n. 5, p. 661-671, 2003. 
[10] FERREIRA, P.M. Uso do geoprocessamento na identificação de áreas de risco para a infestação humana pelo Amblyomma sculptum (Acari: Ixodidae) no município de Piracicaba, SP. 2006, 94 f. Tese (Doutorado em Medicina Veterinária) - Faculdade de Medicina Veterinária e Zootecnia, Universidade de São Paulo, São Paulo, 2006.

[11] FRÖDELL, M. Criteria for achieving efficient contractor-supplier relations. Engineering, Construction and Architectural Management, v. 18, p. 381-393, 2011.

[12] GUGLIELMONE, A.A.; ROBBINS, R.G.; APANASKEVICH, D.A.; PETNEY, T.N.; ESTRADA-PEÑA, A.; HORAK, I.G.; SHAO, R.; BARKER S. The Argasidae, Ixodidae and Nuttalliellidae (Acari: Ixodidae) of the world: a list of valid species names. Zootaxa, v. 2528, p. 1-28, 2010.

[13] ILLOLDI-RANGEL， P.; RIVALDI， C.L.; SISSEL， B.; FRYXELL， R.T.; GORDILLO-PÉREZ， G.; RODRÍGUEZ-MORENO， A.; WILLIAMSON， P.; MONTIEL-PARRA, G.; SÁNCHEZ-CORDERO, V.; SARKAR, S. Species distribution models and ecological suitability analysis for potential tick vectors of Lyme disease in Mexico. Journal Of Tropical Medicine, v. 2012, n. 2012, p. 1-10, 2012.

[14] INSTITUTO BRASILEIRO DE GEOGRAFIA E ESTATÍSTICA (IBGE). Base de informações por setor censitário. Censo demográfico 2010. Resultados do universo, Rio de Janeiro. IBGE, Rio de Janeiro, 2010.

[15] INSTITUTO BRASILEIRO DE GEOGRAFIA E ESTATÍSTICA (IBGE). Censo agropecuário. Disponível em: http://cidades.ibge.gov.br/xtras/temas.php?lang=\& codmun $=330555 \&$ idtema $=3 \&$ search $=$ rio-dejaneiro|seropedica|censoagropecuario 2006 Acesso em 14 de out. 2013.

[16] JAMES, M.C.; BOWMAN, A.S.; FORBES, K.J.; LEWIS, F.; MCLEOD, J.E.; GILBERT, L. Environmental determinants of Ixodes ricinus ticks and the incidence of Borrelia burgdorferi sensu lato, the agent of Lyme borreliosis, in Scotland. Parasitology, v. 140, n. 2, p. 237-246, 2013.

[17] LABRUNA, M.B.; KERBER C.E.; FERREIRA, F.; FACCINI, J.L.H.; WAAL, D.T.; GENNARI, S.M. Risk factors to ticks infestations and their occurrence on horses in the state of São Paulo, Brazil. Veterinary Parasitology, v. 97, n. 1, p. 1-14, 2001.

[18] LAGEOP- Laboratório de Geoprocessamento/UFRJ. Vista Saga 2007. Disponível em: http://www.lageop.ufrj.br/downloads.php Acesso em: 30 jun 2013.

[19] LAMBIN, E.F.; TRAN, A.; VANWAMBEKE, S.O.; LINARD, C.; SOTI, V. Pathogenic landscapes: Interactions between land, people, disease vectors, and their animal hosts. International Journal of Health Geographics, v. 9, n. 54, p. 54-67, 2010.

[20] LIMA, J.P.C.; SANTOS, L.A.F. Floresta nacional Mário Xavier uma proposta de planejamento e de gestão ambiental - justificativa. Floresta e Ambiente, v. 5, n. 1, p. 216-218, 1998.

[21] MALCZEWSKI, J. GIS-based land-use suitability analysis: a critical overview. Progress in Planning, v. 62, n. 1, p. 3-65, 2004. 
[22] MARQUES, E.D.; TUBBS-FILHO, D.; GOMES, O.V.O.; SILVA-FILHO, E.V. Impactos das cavas de extração de areia sobre a química das águas subterrâneas na região da Piranema, bacia sedimentar de Sepetiba, Rio de Janeiro. In. TUBBS-FILHO, D; ANTUNES, J.C.O.; VETTORAZZI, J.S. Bacia hidrográfica dos rios Guandu, da Guarda e Guandu Mirim. Experiências para a gestão dos recursos hídricos. Rio de Janeiro: INEA, 2012, p. 215-237.

[23] MATTOS, C.L.V.; SILVA, M.A.R.; OLIVEIRA, M.N. Boletim agrometeorológico UFRRJ - 1997. Floresta e Ambiente, v. 6, n.1, p. 149-158, 1999.

[24] PADILHA, D.G.; TREVISAN, M.L.; CRUZ, J.C. Sensibilidade do modelo de fragilidades ambientais à ponderação multicriterial: aspectos físicos da bacia hidrográfica do Alto Uruguai. Floresta, v. 44, n. 3, p. 535-548, 2014.

[25] PEDRO, T.B. Estudo dos fatores climáticos e ambientais associados à ocorrência de carrapatos Amblyomma sculptum (Acari: Ixodidae) adultos na mesorregião Metropolitana do Rio de Janeiro. 2015, 40 f. Dissertação (Mestrado em Ciências Veterinárias) - Instituto de Veterinária, Universidade Federal Rural do Rio de Janeiro, Seropédica, 2015.

[26] PEDRO, T.B.; SILVEIRA, A.K.; TASSINARI, W.S. Landscape features associated to the occurrence of Amblyomma sculptum ticks: an application of zero inflated regression models. Revista Brasileira de Medicina Veterinária, v. 38, supl. 3, p. 80-84, 2016.

[27] PETERSON, A.T. Uses and requirements of ecological niche models and related distributional models. Biodiversity Informatics, v. 3, n. 1, p. 59-72, 2006.

[28] QUEIROGAS, V.L.; DEL-ClARO, K.; NASCIMENTO, A.R.T.; SZABÓ, M.P.J. Capybaras and ticks in the urban areas of Uberlândia, Minas Gerais, Brazil: ecological aspects for the epidemiology of tick-borne diseases. Experimental and Applied Acarology, v. 57, n. 1, p. 75-82, 2012.

[29] RANDOLPH, S.E. Ticks ecology: processes and patterns behind the epidemiological risk posed by ixodid ticks as vectors. Parasitology, v. 129, p. 37-65, 2004.

[30] ROSÀ, R; PUGLIESE, A. Effects of tick population dynamics and host densities on the persistence of tick-borne infections. Mathematical Biosciences, v. 208, p. 216-240, 2007.

[31] SCHIMIDT, K.A.; OSTFELD, R.S. Biodiversity and the dilution effect in disease ecology. Ecology, v. 82, p. 609-619, 2001.

[32] SILVEIRA, A.K. Distribuição espacial e flutuação sazonal de carrapatos e modelo geoambiental sobre a favorabilidade de ocorrência de Hydrochoerus hydrochaeris e Amblyomma cajennense. 2014, 76 f. Tese (Doutorado em Ciências Veterinárias) Instituto de Veterinária, Universidade Federal Rural do Rio de Janeiro, Seropédica, 2014.

[33] SILVEIRA, A.K.; FONSECA, A.H. Caracterização de ambientes com potencial para ocorrência de carrapatos transmissores de agentes patogênicos para humanos. Boletim do Parque Nacional do Itatiaia, Itatiaia, v. 13, nov. 2011. Disponível em: http://www.icmbio.gov.br/parnaitatiaia/images/Boletins_de_Pesquisa/bpni_v13.pdf Acesso em: 25 jun. 2013. 
[34] SILVEIRA, A.K.; FONSECA, A.H. Distribuição, diversidade e sazonalidade de carrapatos em ambientes institucionais com diferentes graus de intervenção humana no estado do Rio de Janeiro, Brasil. Revista Brasileira de Medicina Veterinária, v. 35 (supl. 2), p. 1-12, 2013.

[35] SONENSHINE, D.E. Biology of ticks. New York: Oxford University Press, 1993, v. 2, $465 \mathrm{p}$.

[36] SOUZA, C.E.; MORAES-FILHO, J.; OGRZEWALSKA, M.; UCHOA, F.C.; HORTA, M.C.; SOUZA, S.S.L.; BORBA, R.C.M.; LABRUNA, M.B. Experimental infection of capybaras Hydrochoerus hydrochaeris by Rickettsia rickettsii and evaluation of the transmission of the infection to ticks Amblyomma sculptum. Veterinary Parasitology, v. 161, n. 1-2, p. 116-121, 2009.

[37] SZABÓ, M.P.J.; CASTRO, M.B.; RAMOS, H.G.C.; GARCIA, M.V.; CASTAGNOLLI, K.C.; PINTER, A.; VERONEZ, V.A.; MAGALHÃES, G.M.; DUARTE, J.M.B.; LABRUNA, M.B. Species diversity and seasonality of free-living ticks (Acari: Ixodidae) in the natural habitat of wild Marsh deer (Blastocerus dichotomus) in Southeastern Brazil. Veterinary Parasitology, v. 143, p. 147-154, 2007.

[38] SZABÓ, M.P.J.; LABRUNA, M.B.; GARCIA, M.V.; PINTER, A.; CASTAGNOLLI, K.C.; PACHECO, R.C.; CASTRO, M.B.; VERONEZ, V.A.; MAGALHÃES, G.M.; VOGLIOTTI, A.; DUARTE, J.M.B. Ecological aspects of the free-living ticks (Acari: Ixodidae) on animal trails within Atlantic rainforest in south-eastern Brazil. Annals of Tropical Medicine and Parasitology, v. 103, n. 1, p. 57-72, 2009.

[39] VANWAMBEKE, S.O.; SUMILO, D.; BORMANE, A.; LAMBIN, E.F.; RANDOLPH, S.E. Landscape predictors of tick-borne encephalitis in Latvia: land cover, land use, and land ownership. Vector-Borne and Zoonotic Diseases, v. 10, n. 5, p. 497-506, 2010.

[40] VELOSO, H.P.; RANGEL-FILHO, A.L.R.; LIMA, J.C.A. Classificação da Vegetação Brasileira, Adaptada a um Sistema Universal. Rio de Janeiro: Instituto Brasileiro de Geografia e Estatística, 1991, 124 p.

[41] VERONEZ, V.A.; FREITAS, V.B.; OLEGÁRIO, M.M.M.; CARVALHO, W.M.; PASCOLI, G.V.T.; THORGA, K.; GARCIA, M.V.; SZABÓ, M.P.J. Ticks (Acari: Ixodidae) within various phytophysiognomies of a Cerrado reserve in Uberlândia, Minas Gerais, Brazil. Experimental and Applied Acarology, v. 50, n. 2, p. 169-179, 2010.

[42] XAVIER-DA-SILVA, J. Geoprocessamento para análise ambiental. Rio de Janeiro: J. Xavier da Silva, 2001. 228 p.

[43] XAVIER-DA-SILVA, J.; PERSSON, V.G.; LORINI, M.L.; BERGAMO, R.B.A.; RIBEIRO, M.F.; COSTA, A.J.S.T., IERVOLINO, P.; ABDO, O.E. Índices de Geodiversidade: aplicações de SIG em estudos de biodiversidade. In. GARAY, I.; DIAS, B. Conservação da Biodiversidade em Ecossistemas Tropicais. Petrópolis: Vozes, 2001, p. 299-316. 